\title{
EVALUACIÓN DE LAS COMPETENCIAS PARA LA FORMACIÓN TUTORES DE E-LEARNING
}

\author{
(COMPETENCES' EVALUATION FOR THE DEVELOPMENT OF E-LEARNING TUTORS)
}

\author{
Ana María Ehuletche \\ Adriana De Stefano \\ Universidad Nacional de Mar del Plata (Argentina)
}

\section{RESUMEN}

El presente trabajo muestra los primeros avances del proyecto de investigación: Diseño curricular por competencias y criterios de evaluación para la formación de tutores de e-learning.

Los resultados obtenidos por nuestro grupo de investigación específicamente en el proyecto 2005-2007 "Los componentes del diseño pedagógico socioconstructivista: interacción y tutorías. Impacto sobre el logro académico en procesos de enseñanza a distancia”, se incorporan como insumos básicos para el estudio actual. Estos antecedentes permiten plantear la incidencia de los componentes interacción y tutorías, puntualmente la frecuencia y la calidad comunicacional sobre el logro académico y la satisfacción de los alumnos. El reporte mostrará los resultados del análisis de dos cursos equivalentes de la Tecnicatura en Gestión Cultural, en su formato didáctico tecnológico para Campus virtual a partir de intervenciones en los foros con modalidades tutoriales no controladas. Estos hallazgos constituirán una primera etapa para definir las competencias del tutor en la enseñanza on-line.

Palabras clave: competencias tutoriales, e-learning, interacción, componentes, diseño, categorías comunicacionales.

\begin{abstract}
This article reports the initial findings of a research project, "Curricular design for competences and evaluation criteria for the development of e-learning tutors." The results obtained by our research project in 2005-2007, "Components of the socioconstructivist pedagogical design, interaction and tutorials and impact on academic achievement in distance learning," are taken as basic data for the present study. This background permits us to inquire about the incidence of components and the interaction between tutorials, especially their frequency and communication quality, and students' academic achievements and satisfaction. The report includes analysis of two equivalent courses for the technical degree in Cultural Management and the Virtual Campus technological didactic format of
\end{abstract}


discussion forums developments without controlled tutorials. These results are our first step toward defining competencies of on-line tutors.

Keywords: tutorials' competences, e-learning, interaction, compounds, design, communicational categories.

Los estudios previos realizados por el grupo de investigación permitieron analizar: la incidencia de los procesos interaccionales entre los tutores y los alumnos y de los alumnos entre sí. A partir de observar, además, la relación de la producción de este fenómeno con la frecuencia de la participación de los tutores y la calidad de las comunicaciones de éstos y los alumnos producidas en los foros. Esta situación nos llevó a proponer una evaluación por competencias de los diseños curriculares, profundizando la fundamentación psicológica y pedagógica para definir el perfil profesional requerido para la actuación del docente de e-learning.

La revisión de las prácticas docentes desde el marco de referencia de la evaluación por competencias aportará elementos para orientar el diseño de capacitación de tutores para estos entornos.

\section{DESARROLLO}

Nuestra línea de investigación aborda la temática de los diseños pedagógicos en entornos virtuales de enseñanza, focalizando la indagación sobre los componentes interacción y tutoría, su relación con el mejoramiento de la calidad educativa. Los resultados obtenidos nos han señalado que la modalidad de intervención del tutor correlaciona con los logros académicos y satisfacción de los alumnos.

De acuerdo a estas evidencias surge el desafío de la formación de los tutores de e-learning. ${ }^{1}$ La complejidad de esta modalidad educativa implica el desarrollo de múltiples roles en el diseño y producción didáctica y en la gestión del proceso, sin embargo desde nuestra perspectiva nos ocuparemos específicamente del tutor de cuya actuación experta depende los logros del proceso.

El tutor actúa como mediador generando interacciones entre el alumno y el objeto de conocimiento, de los alumnos entre sí y con él, pero además dadas las características del e-learning debe serlo con estas instancias y el entorno. 
La pertinencia y coherencia de una transformación curricular comenta Carlos Marcelo $^{2}$, abarca esta revisión de los saberes que van a ser "enseñados" desde algún marco teórico que dé cuenta de los paradigmas productivos, de los posicionamientos de la educación dentro de los procesos de innovación, de las decisiones que afectan las condiciones materiales de su implementación, y desde la manera en que serán incorporados los nuevos modos tecnológicos en la gestión de los aprendizajes.

En este artículo mostramos resultados preliminares para el análisis de las capacidades que integran las competencias de los tutores virtuales como paso previo para la definición de un perfil de formación, entendiendo las tutorías como un modo de intervención pedagógica favorecedor de un proceso de comunicación interactiva y donde el rol del tutor es orientar y reorientar los procesos de comprensión y transferencia.

Los nuevos códigos de comunicación establecidos a partir de una mayor difusión de los medios informáticos, generan un lenguaje cotidiano no siempre compartido entre alumnos y docentes. Así también, la presencia de la tecnología deja su impronta en la manera como se gestiona el conocimiento: las posibilidades concretas de las redes, el aprendizaje colaborativo, el uso de la telemática, los fenómenos comunicacionales en los entornos virtuales.

Nuestro estudio toma como referencia para el abordaje el Proyecto Tuning en su línea de trabajo de enfoques de enseñanza con aprendizaje y evaluación de las competencias. El mismo considera que “...las competencias representan una combinación dinámica de conocimiento, comprensión, capacidades y habilidades. Fomentar las competencias es el objeto de los programas educativos. Las competencias se forman en varias unidades del curso y son evaluadas en diferentes etapas. Pueden estar divididas en competencias relacionadas con un área de conocimiento (específicas de un campo de estudio) y competencias genéricas (comunes para diferentes cursos)"3.

La adquisición de competencias en torno a un área profesional se desenvuelve en un itinerario atravesado por redes de empleos, movilidades y formación continua, es decir en trayectorias que implican una combinación de educación formal, aprendizaje en el trabajo y eventualmente educación no formal. Específicamente Gallart $(2003)^{4}$ plantea que el desafío para los sistemas educativos es llegar a formar a las sucesivas cohortes de jóvenes en un nivel aceptable de competencias que se impartan en el sistema formal o cubiertas por políticas de formación no formal. Destacando que las competencias, son la base de la capacitación y 
del aprendizaje en el trabajo y que los cambios continuos en la tecnología y en la organización del trabajo cuestionan la rigidez de los sistemas de formación dirigidos a ocupaciones específicas y tiende a poner cada vez más el énfasis en las competencias de empleabilidad correspondientes a la formación general.

Desde este enfoque el concepto de competencia no se asimila únicamente al desempeño laboral ni tampoco a la adquisición de conocimientos prácticos (saber hacer), representa una red conceptual amplia que abarca un conjunto de capacidades desarrollables a través de aprendizajes con distintos niveles de complejidad. Así, podemos reconocer competencias básicas o fundamentales, genéricas o comunes, específicas o especializadas y laborales.

\section{PERFIL PROFESIONAL}

La determinación de las áreas de competencia es el primer nivel de especificación del perfil profesional. Partiendo del análisis del campo profesional, se recortan las grandes áreas de la actividad tutorial. En nuestro caso está claramente definido por el nivel, la modalidad y el encuadre institucional. Son elementos de las áreas de competencia: los desempeños complejos que involucran y movilizan capacidades transferibles a diversos contextos de enseñanza; y los estándares a partir de los cuales la actividad del tutor es evaluada como competente en una situación real de trabajo.

Los últimos avances en nuestro grupo de investigación, reflejaron que la presencia del tutor y la frecuencia de las interacciones constituyen los componentes de mayor significatividad en la propuesta pedagógica diseñada e implementada. Situación esta, que se ve avalada por diferentes autores que sostienen la efectividad de los procesos interaccionales enriqueciendo los contextos virtuales, enfatizando el diálogo educativo, determinando niveles de interacción (Simonson, 2000; Mac Vay, 2002; Garrison y Anderson, 2003; Barberá, 2004).

La experiencia mostró que el actuar mediado electrónicamente del tutor, requiere del mismo, presencias mas frecuentes, ya que debe gestionar interacciones y aportes constantes de los alumnos para guiar la construcción de textos enriquecidos. Esto permitirá construir un nivel de visibilidad y por lo tanto de presencia social contrarrestando la debilidad por la asincronía del medio, marcando las diferencias entre contextos virtuales en lo que hace a la construcción del otro como más cercano. Esta situación constituye una ayuda pedagógica que potencia la permanencia del alumno en el curso evitando los riesgos de deserción. Desde esta perspectiva estamos 
hablando de procesos colaborativos en Educación a Distancia; por lo tanto estamos obligados a incluir lo tecnológico dentro del contexto además del factor humano, planteamiento que sugiere algunas modificaciones sobre la función del tutor, tales como:

- La necesidad de convertirse en un intermediario entre el estudianteylainstitución, aportando información y asesoramiento al sistema, pues la credibilidad en el mismo inicialmente pasará por él.

- Pasar de ser un experto en contenidos a un facilitador del aprendizaje, a partir de diseños de experiencias que le ofrezcan al estudiante una estructura inicial para la generación de la interacción.

- Poseer mínimas habilidades técnicas no sólo para intervenir en el sistema, sino para resolver las limitaciones que se le vayan presentando al estudiante para interaccionar en el mismo.

- Presentar los contenidos de los materiales de manera tal que favorezca al máximo el aprendizaje cuando su uso sea individual y solitario, a través de diseños específicos, de una organización lógica de los contenidos y de inserciones de bibliografía, resúmenes, mapas conceptuales, definiciones, esquemas, referencias a páginas Web y actividades.

- Potenciar el feedback informativo mediante el envío de mensajes por campus virtual a partir de comentarios planteados por los estudiantes, tales como reflexiones que surjan de la revisión de trabajos, aclaración de posibles dudas y orientando antes del encuentro con dificultades.

- Plantear tareas de enseñanza-aprendizaje abiertas y auténticas, que consigan activar en los estudiantes tanto sus conocimientos previos y sus procesos cognitivos como la organización y elaboración del conocimiento. El planteamiento de estas actividades ha de ir acompañado de suficiente información para que el estudiante pueda realizarla sin tener que depender excesivamente del profesor. En caso contrario éste deberá abrir las vías de comunicación telemáticas para que los estudiantes puedan compartir con él, los objetivos y las condiciones de la tarea. 
- Proporcionar recursos suficientes a los estudiantes desde materiales en diferentes formatos hasta enlaces a páginas web, a fin de que puedan responder a las demandas que se realicen, activando procesos cognitivos de aprendizajes adecuados.

- Proponer un proceso de evaluación de los aprendizajes que ponga de manifiesto el resultado de la construcción de conocimiento por parte del estudiante.

- Establecer una cantidad muy importante de interacciones con los estudiantes para recoger información sobre el proceso y la calidad de la construcción de conocimientos que se vayan produciendo. Estos indicadores deberán dar una perspectiva suficientemente amplia al profesor sobre el proceso de aprendizaje, a fin de que pueda tomar la iniciativa y utilizar metodologías y técnicas instruccionales para dar soporte adecuado a cada proceso de aprendizaje que sigue cada estudiante.

- Desplegar un mayor número de ayudas y ver que estas sean de diferente naturaleza, lo que supone una mayor complejidad en su actividad docente, ya que a menudo deberá pensar y desarrollar variadas maneras de presentar el mismo contenido y utilizar estratégicamente varios dispositivos semióticos, en función de las necesidades de los estudiantes.

- Estar preparado para discontinuidades en el aprendizaje de los estudiantes y a diferencias entre ellos más notables que en las aulas presenciales. En el contexto presencial la figura del profesor y la instrucción única homogeniza. En la educación a distancia en cambio, puede haber más fuentes de acceso a la información y, por lo tanto, más posibilidades de divergencia en los procesos de aprendizaje. $^{5}$

- La influencia del tutor en la gestión del conocimiento debe incluir otros factores que potencien la construcción del conocimiento, incluyendo pautas interaccionales que desplieguen en los estudiantes los procesos metacognitivos individuales y grupales propios del entorno.

\section{MÉTODOS Y TÉCNICAS EMPLEADOS}

A través de un diseño exploratorio se analizaron en esta etapa las intervenciones de los tutores y los alumnos. Para este reporte de la población total ${ }^{6}$ se toma la cohorte 2007. Se trabajó con tres asignaturas de la carrera, dos pertenecientes a 
primer año y una a segundo :1- Historia Cultural del Siglo XX ( $1^{\circ} a$ ño), $N=165$; 2Políticas Culturales ( $1^{\circ}$ año) $\mathrm{N}=161$; 2- Historia y Teoría de la Cultura de ( $\left.2^{\circ} a n ̃ o\right)$, $\mathrm{N}=65$. Número total de casos: 391 .

\section{Instrumentos}

- Escala tipo Likert de satisfacción de los alumnos.

- Reporte de participación (alumno y tutor) en las distintas secciones del aula virtual.

- Entrevista a tutores y coordinadores.

\section{Categorías comunicacionales}

En el análisis de las interacciones en el foro se aplicó un instrumento que clasifica las categorías comunicacionales, Análisis de las Categorías Comunicacionales (Ehuletche y Terroni, 2002).

Durante el proceso de análisis se registraron tanto la cantidad de intervenciones en el foro, (CI) producida por los miembros del grupo, como las unidades comunicativas (UC) contenidas en cada una ellas.

Las categorías comunicacionales que se observaron se clasificaron de acuerdo al instrumento en:

- Conceptuales: refieren a los mensajes cuyo contenido apunta a la construcción de conocimiento en la temática, haciendo referencia a dudas, opiniones, elaboraciones, cuestionamientos, etc.

- Socioemocionales: denotan actitudes, valores, emociones, sentimientos y otros componentes que hacen al clima social del grupo.

- Gestión de Tarea: aluden a cuestiones organizativas y operativas, uso de la herramienta informática, consultas sobre dificultades tecnológicas, etc.

En esta etapa se muestran sólo los resultados del reporte de participación alumno/tutor en los foros y del análisis del instrumento categorías comunicacionales. 


\section{RESULTADOS}

En la misma línea que los hallazgos anteriores para nuestro grupo de investigación, los resultados de esta primera etapa muestran la relación entre la frecuencia de intervención del tutor, la de los alumnos, el logro académico y la comunicación en cuanto a las categorías presentes en el texto para las interacciones en los foros. Si observamos los datos de la Tabla 1 podemos comparar la cuantificación de las variables para los tres cursos estudiados. Así, para Políticas Culturales el promedio de notas a través de las cuales se midió el logro académico $(8,23)$ en Historia y Teoría de la Cultura $(7,40)$ y en Historia Cultural $(6,85)$. La relación de este promedio se da con la frecuencia de intervenciones en el foro de alumnos y tutores así para la primer materia señalada es de 386 intervenciones, para la segunda 98 y la tercera 168 , la frecuencia de intervenciones del tutor 28,75 para la de mejor promedio, 18,36 para el segundo y 20,23 para el tercero. Además vemos la frecuencia mas alta de intervención de los alumnos para la asignatura de mejor promedio., como también lo es la más alta relación de intervenciones tutor por alumno o,69.

En cuanto a las categorías comunicacionales en el curso de mayor promedio se da una mayor frecuencia de socioemocionales $(38,09 \%)$ que sumadas a la gestión de tarea $(31,29 \%)$, suman 69,38 , siendo el puntaje más alto en relación a los otros dos cursos. Esto se refuerza al cruzar los resultados del promedio del curso de más bajo promedio $(6,85)$ con la más alta frecuencia de categorías comunicacionales conceptuales.

\section{CONCLUSIONES}

Los datos obtenidos parecen corroborar que el tutor de e-learning requiere habilidades para presencias mas frecuentes, para gestionar interacciones y aportes constantes a los alumnos, para guiar la construcción de textos enriquecidos, para gestionar los circuitos feedback, para sostener la ausencia de presencia social con textos enriquecidos con comunicaciones socioemocionales. Situaciones estas sobre todo la en relación a la ausencia del otro, que pone en riesgo la permanencia del alumno, si no se puede construir un nivel de visibilidad y por lo tanto de presencia social contra restando la debilidad por la asincronía del medio, marcando las diferencias entre contextos virtuales en lo que hace a la construcción de ese otro como mas cercano. El tutor debe tener competencias que lo habiliten para generar una ayuda pedagógica que potencia la permanencia del alumno en el curso evitando los riesgos de deserción, aumente su satisfacción, potencie los logros académicos, además debe ser hábil en el manejo tecnológico que permita un buen uso de los recursos y materiales como sortear imprevistos. 
A. EHULetche; A. De STEFAno

Evaluación De Las Competencias Para La Formación Tutores De E-Learning

\begin{tabular}{|c|c|c|c|c|}
\hline \multirow{3}{*}{ 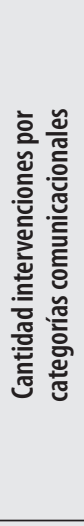 } & 竎 & $\begin{array}{l}0 \\
\infty \\
\infty \\
\infty \\
\infty \\
\infty \\
\dot{f} \\
f\end{array}$ & 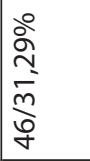 & 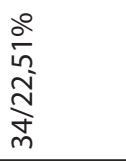 \\
\hline & 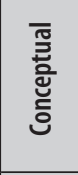 & 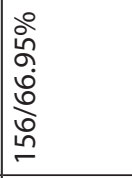 & $\begin{array}{l}0 \\
\frac{0}{6} \\
0 \\
m \\
\frac{m}{\sigma}\end{array}$ & 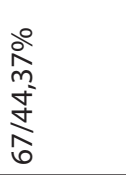 \\
\hline & 을 & 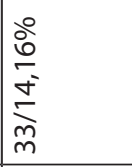 & 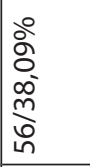 & 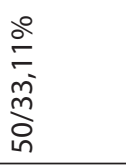 \\
\hline \multicolumn{2}{|c|}{ 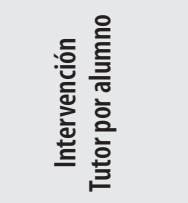 } & $\overline{\widetilde{N}}$ & O̊) & 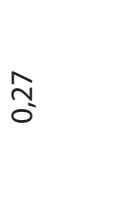 \\
\hline \multirow{2}{*}{ 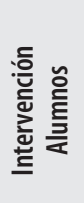 } & 운 & $\begin{array}{l}\frac{0}{2} \\
2 \\
1\end{array}$ & $\begin{array}{l}8 \\
0 \\
0\end{array}$ & 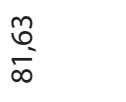 \\
\hline & ч & $\stackrel{\vec{m}}{m}$ & $\stackrel{\stackrel{n}{\sim}}{\sim}$ & $\infty$ \\
\hline \multirow{2}{*}{ 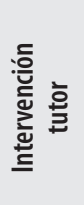 } & 운 & $\begin{array}{l}\stackrel{n}{ } \\
\stackrel{N}{N}\end{array}$ & $\stackrel{\substack{n \\
\infty \\
\infty}}{\stackrel{0}{\infty}}$ & $\begin{array}{l}\stackrel{\infty}{m} \\
\infty^{-}\end{array}$ \\
\hline & ᄂ & m & $\mp$ & $\stackrel{\infty}{-}$ \\
\hline \multicolumn{2}{|c|}{ 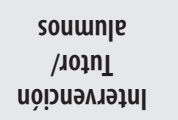 } & $\underset{0}{\infty}$ & $\begin{array}{l}\infty \\
m\end{array}$ & 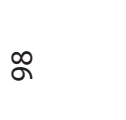 \\
\hline \multicolumn{2}{|c|}{$\begin{array}{l}\text { setou } \\
\text { o!pəumold }\end{array}$} & $\begin{array}{l}n \\
\infty \\
0 \\
0\end{array}$ & $\underset{\infty}{\stackrel{\sim}{\infty}}$ & 茎 \\
\hline \multicolumn{2}{|c|}{ eגnı̨eufisy } & 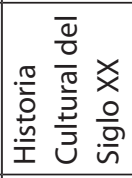 & 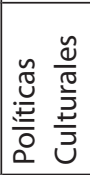 & 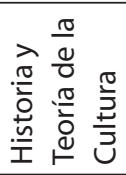 \\
\hline \multicolumn{2}{|c|}{ OSE) } & - & $\sim$ & $m$ \\
\hline
\end{tabular}




\section{NOTAS}

$1 \quad$ A partir de la explosión tecnológica de las TICs y su utilización acrítica en los procesos de enseñanza aprendizaje los resultados evaluados a partir de los logros obtenidos imponen la necesidad de una revisión de las prácticas pedagógicas.

2 Marcelo, C. (2005). Estudio sobre competencias profesionales para e-learning. Proyecto Prometeo El Portal de e-Learning para la Formación Profesional Ocupacional es el resultado de un esfuerzo continuado por parte de la Dirección General de Formación Profesional Ocupacional y de los miembros del Grupo de Investigación IDEA de la Universidad de Sevilla, a través del Proyecto Prometeo. Bajo la dirección de Carlos Marcelo García, se han venido desarrollando una amplia variedad de acciones que han impulsado la utilización de las nuevas tecnologías en la formación de teleformadores, diseño de materiales multimedia, difusión del e-Learning, creación de redes de formadores, etc.

3 Informe final Proyecto Tuning América Latina, 2004-2007. [en línea] Disponible en: http://tuning.unideusto.org/tuningal/ (consulta 2008, 29 de agosto).

4 Gallart, M. (2003). La formación para el trabajo y los jóvenes en América Latina. En el marco del proyecto "Formación Técnica y Profesional en América Latina", implementada por la CEPAL y la Sociedad Alemana de Cooperación Técnica (GTZ). Santiago de Chile.

5 Ehuletche, A.; De Stefano, A.; Banno, B.; González, E. (2007). Ajustes pedagógicos en entornos virtuales: los procesos colaborativos. EDUTEC/2007. Buenos Aires.

6 Los alumnos de las cohortes 2007/2008/2009 de la Tecnicatura en Gestión Cultural dictada por el Sistema de Educación Abierta y Distancia de la UNMdP. 


\section{REFERENCIAS BIBLIOGRÁFICAS}

Banno, B. (2005). Diseños curriculares por competencias, Boletín Educativo. ISSN 0328-3534, Año 17 - No 178 . Ediciones Novedades Educativas, (15-17).

Braslavsky, C. (1994). Una función de la escuela: formar sujetos activos en la construcción de su identidad y de la identidad nacional. En: Filmus (comp.), Para qué sirve la escuela. Buenos Aires: Grupo Editorial Norma.

Chiavenato, I. (1995). Administración de Recursos Humanos. México: Editorial MacGraw-Hill.

Collison, G.; Elbaum, B.; Haavind, S.; Tinker, R. (2000). Facilitating on line learning. Effective strategies for moderators. USA: Atwood Publishing.

Ehuletche, A.; Terroni,N.(2002). Elaboración de categorías de comunicación en las interacciones colaborativas. Revista del Instituto Rosario de Investigaciones en Ciencias de la Educación. CONICETUNR. Rosario, Argentina.

Ehuletche, A.; Banno, B.; Del Río, M. J. A.; Elger, S. (2003). La evaluación del soporte tutorial en la mediación electrónica desde la perspectiva de la satisfacción del alumno. RIED, Revista Iberoamericana de Educación a Distancia, 6 (1). (91-106).

Gallart, M. (2003). La formación para el trabajo y los jóvenes en América Latina. En el marco del proyecto "Formación Técnica y Profesional en América Latina", implementada por la CEPAL y la Sociedad. [en línea] Disponible en: http://cvc.cervantes.es/ obref/formacion virtual/formacion continua/peiro.htm (consulta 2007, 19 de septiembre).

Litwin, E. (2000). La educación a distancia. Temas para el debate en una nueva agenda educativa. Buenos Aires: Amorrortu.

Marcelo, C. (2005). Estudio sobre competencias profesionales para e-learning. Proyecto Prometeo. Santiago de Chile: Agencia Alemana de Cooperación Técnica (GTZ).

Peiró, J. (2006). Las competencias en la sociedad de la información: nuevos modelos formativos. [en línea] Disponible en: http://cvc.cervantes.es/ obref/formacion virtual/formacion continua/peiro.htm (consulta 2007, 19 de septiembre).

Perrenoud, P. (2007). Diez Nuevas competencias para enseñar. Barcelona, España: Editorial GRAO.

Proyecto Tuning América Latina. (2007). Informe final: Reflexiones y perspectivas de la Educación Superior en América Latina. [en línea] Disponible en: http:// www.tuning.unideusto.org/tuningal/ (consulta 2007, 19 de septiembre).

Santangelo, H. (2000). Modelos pedagógicos en los Sistemas de Enseñanza no presencial basados en Nuevas tecnologías y Redes de comunicación. Revista de la $O E I, 24$. Septiembre-diciembre. en línea] Disponible en: http://www.campusei.org/revista/rie24a06.htm (consulta 2007, 19 de septiembre). 


\title{
PERFIL ACADÉMICO Y PROFESIONAL DE LA AUTORAS
}

Ana María Ehuletche. Docente de la cátedra de Psicología Educacional en la Facultad se Psicología de la UNMdP y en la Universidad Atlántica Argentina. Directora del proyecto de investigación sobre la temática Diseño curricular por competencias y criterios de evaluación para la formación de tutores de e-learning. Participación en Congresos, publicaciones en revistas con referato y capítulo de libros referidas a diseños pedagógicos en entornos virtuales y las competencias del tutor, enfatizando la construcción de la presencia social.

E-mail: ehulet@mdp.edu.ar

DIRECCIÓN DE LA AUTORA:

\author{
Funes 3350 (7600) \\ Mar del Plata, Argentina
}

Adriana Alicia De Stefano. Me desempeño desde el año 1984 en el Sistema de Educación Abierta y a Distancia (SEAD) como responsable de diseño, producción e implementación de distintas ofertas presenciales, semipresenciales, a distancia y e-learning y Webmaster de la plataforma. Actividades de investigación relacionadas con las problemáticas de la comunicación mediada, interacción en entornos virtuales y competencias tutoriales. Proyecto actual: Diseño curricular por competencias y criterios de evaluación para la formación de tutores de e-learning” (2008/2009).

E-mail: destefan@mdp.edu.ar

DIRECCIÓN DE LA AUTORA:

Matheu 4098 (7600)

Mar del Plata, Argentina

Fecha de recepción del artículo: 31/03/10

Fecha de aceptación del artículo: 16/10/10 